\title{
Didaktik und Methodik des frühen Fremdsprachenlernens im Kindergarten
}

\author{
Patricia Nauwerck \\ Pädagogische Hochschule Schwäbisch Gmünd
}

\begin{abstract}
Zugleich werden verschiedene Konzeptionen des frühen Fremdsprachenlernens sowie deren sprachwissenschaftliche und sprachdidaktische Prämissen vorgestellt. Im Kontext der Gelingensbedingungen für das frühe Fremdsprachenlernen im Kindergarten werden außerdem methodische Aspekte beleuchtet. Darüber hinaus thematisiert der Beitrag, welche Kompetenzen pädagogisches Fachpersonal benötigt, um frühe Fremdsprachenangebote im Vorschulbereich effizient und kindgerecht zu gestalten.
\end{abstract}

Key-words: frühkindliche Lernwege, Spracherwerbs-/Sprachlernprozesse, Konzeptionen des frühen Fremdsprachenlernens, Fremdsprachenlernen im Kindergarten, Kompetenzen

\section{Ausgangslage}

Frühe Fremdsprachenprogramme erfreuen sich in Deutschland seit längerem einer großen Nachfrage. Angesichts dieser Popularität ist es kaum vorstellbar, dass die bilinguale Vorschulerziehung vor noch gar nicht allzu langer Zeit ein weißer Fleck auf der Wissenschaftslandkarte war. Erst im Kontext der internationalen Schulleistungsstudien und dem damit einhergehenden „PISA-Schock“ rückte der Elementarbereich in den Fokus der Bildungspolitik und der Forschung. Seither haben sich sowohl das Selbstverständnis als auch die öffentliche Wahrnehmung des Kindergartens grundlegend verändert: Als Teil des Bildungssystems grenzt sich der Kindergarten inzwischen nicht mehr als eigener Gegenstandsbereich (als „Schonraum“, in dem vor allem Betreuung, Spielen, Basteln und soziales Lernen im Vordergrund stehen) von der Schule ab. Heute liegt der Fokus auf frühkindlichen Bildungsprozessen, die Vorläuferfertigkeiten für das schulische Lernen anbahnen und somit zu einem gelingenden Übergang zwischen den Institutionen - d.h. im Idealfall zu einer durchgängigen, erfolgreichen Bildungsbiographie aller Kinder - beitragen sollen. Darin nimmt das Entwicklungsfeld Sprache eine prominente Stellung ein, denn die sprachlichen Fähigkeiten von Kindern beeinflussen maßgeblich das Gelingen des schulischen Lernens, bei dem die Sprache als Unterrichtsmedium fächerübergreifend eine Schlüsselrolle spielt.

Zudem legitimieren jüngste Forschungsergebnisse u.a. aus der Entwicklungspsychologie sowie den Kognitions- und Neurowissenschaften die Neuorientierung der frühkindlichen Bildung: Anders als lange Zeit angenommen, entwickeln bereits Kleinkinder erste Voraussetzungen für intentionale Lernprozesse, d.h. strategische, selbstregulative und metakognitive Fähigkeiten (Stern et al., 2005. 53.). Aus solchen und anderen Befunden ${ }^{1}$ zur Entwicklung des kindlichen Denkens und Lernens ist deutlich geworden, auf welche Weise Kinder kognitive Operationen in Abhängigkeit von ihrem Weltwissen und ihren Erfahrungen durchführen. Dieses Potenzial kommt beim

\footnotetext{
1 Siehe auch bereichsspezifisches Wissen, Neo-Nativismus (Stern et al., 2005, 54.).
} 
ein- und mehrsprachigen Spracherwerb u.a. über die Sprachbewusstheit ${ }^{2}$ (Sprache und ihre Einheiten fokussieren, mit Sprache spielen, Nachdenken über Sprache, subjektive Theorien/bootstrapping, Metasprache) zum Tragen. Nachweislich sind bereits Kleinkinder in der Lage, mit Sprache zu spielen, über Sprache nachzudenken und dies ggf. auch zu explizieren (vgl. Wehr, 2001; Nauwerck, 2005; Nauwerck, 2013). Mehrsprachige scheinen dabei sogar im Vorteil zu sein (Oomen-Welke, 2003).

Aus dieser veränderten Wahrnehmung kindlicher Kompetenzen leiten sich Ansätze für eine gezielte, möglichst früh einsetzende Förderung ab, der nun nicht länger der Makel vermeintlicher Überforderung anhaftet. Damit öffnete sich nach und auch der bis dato von einem monolingualen Habitus geprägte Kindergarten Bereichen, die dort aus dem damaligen Selbstverständnis heraus ganz bewusst - u.a. aus Sorge vor möglicher „Überforderung“ - ausgegrenzt wurden: der Schriftlichkeit und der Mehrsprachigkeit. Inzwischen hat sich das frühe Fremdsprachenlernen im Vorschulbereich etabliert. Seit die Sprachlehrforschung zu Beginn der 2000Jahre begann, sich dem Frühbereich zuzuwenden, wurden in der Didaktik der bilingualen Vorschulerziehung beachtliche Fortschritte erzielt. Ihre theoretischen Grundlagen wurden gefestigt und stetig ausgebaut, was sich in einer Vielzahl an Publikationen, Materialien und Handreichungen zur Fremdsprachenarbeit im Kindergarten niederschlägt. Die die empirischen Befunde sind ebenso ermutigend wie die praktischen Erfahrungen, die aus den Vorschuleinrichtungen zurückgemeldet werden: Mehrere Studien haben sprachliche Effekte des frühen Fremdsprachenlernens überprüft und übereinstimmend die Wirksamkeit - bezogen auf Sprachverständnis, Sprachproduktion, Wortschatz, Grammatik, Sprachaufmerksamkeit, metalinguistische Fähigkeiten, Sprachlernstrategien nachgewiesen (Wode, 2009; Kersten, 2010a; Kersten, 2010b; Häckel \& Piske, 2011; Nauwerck, 2012). Die Forschungsergebnisse schlagen sich inzwischen zunehmend in den Materialien nieder, welche die Arbeit in den Kindertagesstätten prägen. Die dort tätigen Erzieherinnen und Erzieher verwenden eine Fülle von Texten und Praktiken zur sprachlichen und interkulturellen Förderung der Kinder, in der die gesprochene und die literate Sprache Berücksichtigung finden. Neben fremdsprachlichen Spielen, Liedern, Reimen und Tänzen haben auch Gedichte und Geschichten, Sprachübungen, Rätsel, Rezepte, Sprichwörter Eingang in die bilinguale Vorschulerziehung gefunden.

Der vorliegende Beitrag widmet sich zunächst der Lernerperspektive, indem frühkindliche Lernwege skizziert und aus linguistischer Perspektive Spracherwerbs-/ Sprachlernprozesse modelliert werden.

Zugleich werden verschiedene Konzeptionen des frühen Fremdsprachenlernens sowie deren sprachwissenschaftliche und sprachdidaktische Prämissen vorgestellt. Im Kontext der Gelingensbedingungen für das frühe Fremdsprachenlernen im Kindergarten werden außerdem methodische Aspekte beleuchtet. Darüber hinaus thematisiert der Beitrag, welche Kompetenzen pädagogisches Fachpersonal benötigt, um frühe Fremdsprachenangebote im Vorschulbereich effizient und kindgerecht zu gestalten.

\section{Die Lernerperspektive}

Schon lange gilt es als zweifelsfrei erwiesen, dass Kinder mit mehr als einer Sprache aufwachsen können. In diese Richtung deuten auch bisherige Ergebnisse aus der Sprachlehrforschung. Sie bestätigen, dass das frühe Fremdsprachenlernen bei stim-

2 Zur Metakognition siehe auch Stern et al., 2005, 63ff. 
migen Rahmenbedingungen keine Überforderung darstellt. Piske und Häckel (2011) haben das Fremdsprachenlernen unter Immersionsbedingungen untersucht und sind dabei zu folgenden Befunden gelangt:

a) In Abhängigkeit von Kontaktdauer und Inputstruktur der angebotenen Fremdsprache wurden bei den Vorschulkindern signifikante Zuwächse im Bereich des Lexikons und der Grammatik festgestellt.

b) Eine Abhängigkeit von Geschlecht oder Migrationshintergrund spiegelte sich in den Untersuchungsergebnissen zur fremdsprachlichen Entwicklung nicht wider, wohl aber der Altersfaktor (bezogen auf Grammatik).

c) Frühes Fremdsprachenlernen im Kindergarten wirkt sich nicht negativ auf den Erstspracherwerb (bzw. bei Kindern mit Migrationshintergrund auf Zweitspracherwerb Deutsch) aus.

d) Ergebnisse von Sprachstandserhebungen deuten darauf hin, dass sich frühes Fremdsprachenlernen vielmehr positiv auf die erstsprachliche Sprachentwicklung der Kinder ausgewirkt hat.

Je nachdem, wann das fremdsprachliche Angebot im Kindergarten einsetzt, befinden sich die jungen Lerner - mit Blick auf ihr Alter und die Festigung der Erstsprache - an der Schnittstelle zwischen einem simultanen und frühen sukzessiven Bilinguismus. Daraus ergeben sich, bezogen auf die Sprachverarbeitungsstrategien und das Zusammenspiel von L1 und L2, spezifische Potentiale für das Fremdsprachenlernen, die es gewinnbringend zu nutzen gilt.

Dazu gehört auch, die Lern- und Denkwege der Kindergartenkinder in den Blick zu nehmen: Kinder sind nämlich aktive Lerner, die sich ihr Wissen in der Auseinandersetzung mit ihrer Umwelt konstruieren. Dafür benötigen sie ein anregungsreiches Umfeld und Erwachsene für die Ko-Konstruktion. Denn Kinder lernen mit allen Sinnen, durch Beobachten, Zuhören, Imitieren und in sozialen Interaktionen.

Gleichwohl ist es ein Irrtum anzunehmen, dass die jungen Lerner nur eine einfache Sprache wie Farben, Zahlen, Kinderreime, Lieder lernen wollen: „Children need more than ,simple' language in the sense that only , simple' topics are covered (Cameron, 2001).

Deshalb brauchen sie zum Ausbau der zweiten Sprache neben kompetenten Sprachvorbildern auch einen differenzierten Input und ein sprachanregendes Umfeld. Lynne Cameron bringt es auf den Punkt, wenn sie feststellt, dass

"Children do have a less complicated view of the world than older children or adults, but this fact does not imply that teaching children is simple or straightforward. On the contrary, the teacher of children needs to be highly skilled to reach into children's worlds (...)."

\section{Konzeptionen des frühen Fremdsprachenlernens}

Beim frühen Fremdsprachenlernen im Kindergarten stehen sich - je nach Organisationsform, Art und Umfang des Sprachangebots und den damit verbundenen Zielsetzungen - zwei übergeordnete Ansätze gegenüber: Das Immersionsmodell („Sprachbad“) und das zeitlich begrenzte, auf Sprachsensibilisierung angelegte Angebotsmodell.

Das Immersionsmodell - mit dem Bildungsziel Zweisprachigkeit anzubahnen - basiert auf Erkenntnissen der Bilinguismusforschung. Methodisch wird nach verschiedenen Prinzipien der funktionalen Sprachentrennung gearbeitet. Beim Zweisprachenmodell wird das Rollenprinzip „une personne - une langue“ wirksam, d.h. die Bezugspersonen der Kinder sind in der Regel Muttersprachler und somit zugleich die 
Vertreter der jeweiligen Sprache und Kultur. Durch die Zusammenarbeit einer deutschen und einer französischen Erzieherin innerhalb einer Kindergartengruppe entsteht eine authentische Situation, die der familiären Zweisprachigkeitserziehung, z.B. in binationalen Ehen, ähnlich ist.

Bei diversen Angebotsansätzen bilingualer Bildung im Kindergarten wird die französische Sprache zeitlich begrenzt in gezielte Aktivitäten des Kindergartenalltags eingebunden. Auf organisatorischer Ebene ist hier zwischen dem Angebot innerhalb der Gruppe und einem gruppenübergreifenden Angebotsmodell zu unterscheiden.

Beim Angebot innerhalb der Gruppe trägt die Erzieherin den Kindern z.B. im Stuhlkreis französische Lieder, Reime, Finger- oder Rollenspiele an. Beim zweiten gruppenübergreifenden - Modell finden regelmäßig für einen Teil der Kinder aus mehreren Gruppen (z.B. alle Schulanfänger) spezielle fremdsprachliche Aktivitäten statt, bei dem die Kinder die andere Sprache und Kultur kennenlernen können.

Die Unterscheidung zwischen Immersions- und Angebotsansatz ist vor allem mit Blick auf die damit verbundenen Ziele wichtig: Schließlich dürfen bei allen Beteiligten insbesondere in Bezug auf den sprachlichen Ertrag keine falschen Erwartungen geweckt werden, die am Ende sowohl bei den Kindern als auch den Erzieherinnen zu Motivationsverlust führen können. Da es heute als wissenschaftlich belegt gilt, dass neben der Qualität insbesondere auch die Quantität des zweitsprachlichen Inputs über das Ergebnis der Fremdsprachenvermittlung entscheidet, kann ein zeitlich sehr begrenztes (i.d.R. 20minütiges) Französischangebot einmal in der Woche zwangsläufig nicht den gleichen sprachlichen Effekt herbeiführen wie das tägliche „Sprachbad“. Während das „Sprachbad“ mit zwei authentischen Sprachmodellen auch den jeweiligen soziokulturellen Hintergrund einschließt, kann dieser bei einem zeitlich begrenzten frankophonen Angebot durch eine deutsche Erzieherin nur in kleinen Ausschnitten gezeigt werden, wenn z.B. Lieder, Reime, Fingerspiele, Sprachrituale (Begrüßung, Verabschiedung, Frage oder Auskunft zum aktuellen persönlichen Befinden) beinhalten. Beim Angebotsansatz werden jene Sprachelemente zudem meist ganzheitlich-imitativ vermittelt, während sie unter Immersionsbedingungen als Teil authentischer Alltagskommunikation auf semantischer, syntaktischer und pragmatischer Ebene Anwendung finden.

Bei den Angebotsansätzen stehen aus Sicht der Erzieherinnen vor allem pädagogische Zielsetzungen wie z.B. Ausbildung von Toleranz, Freude im Umgang mit der anderen Sprache, im Vordergrund. Gleichwohl liegen Studienergebnisse vor, die darauf hindeuten, dass der Umfang des Sprachangebots offenbar ausreicht, um die Kinder an den Sprachklang heranzuführen, ihr Interesse an der anderen Sprache und Kultur wecken und damit positive Einstiegsvoraussetzungen für das weitere Sprachenlernen zu bewirken. Somit haben die Angebotsansätze neben den Immersionsmodellen durchaus ihre Berechtigung (Nauwerck, 2012). In der Fachliteratur wird „künstlicher Zweisprachigkeit" (etwa in einem einsprachigen Elternhaus) wenig Aussicht auf Erfolg eingeräumt. Aus dieser Perspektive macht es für Kindergärten mit monolingualer Personalkonstellation bzw. bei begrenzten Französischkenntnissen der Erzieherinnen mehr Sinn, in einem Angebotsansatz durch ein überschaubares Repertoire von Liedern, Reimen, Fingerspielen bei den Kindern eine solide Artikulationsbasis zu schaffen als mit aufgesetzter Immersion am Ende keine oder sehr fragwürdige Resultate zu erzielen.

Unter Bezug auf vorliegende sprachwissenschaftliche und sprachdidaktische Erkenntnisse hatte die o.g. Studie im Kontext des Freiburger Projekts „Bilinguale Bildung - Französisch im Kindergarten“ - das Ziel, Chancen und Grenzen früher Fremdsprachenvermittlung im Kindergarten auszuloten. Das zentrale Interesse war darauf 
ausgerichtet, unter welchen Bedingungen (organisatorischen, inhaltlichen und methodischen Gegebenheiten) sich das Erlernen einer weiteren Sprache hier bei Vorschulkindern als besonders effizient erweist.

Maßstab des Gelingens waren dabei die zweitsprachlichen Kenntnisse und Fertigkeiten, die die Kinder in den verschiedenen Organisationsformen sowie den damit verbundenen Sprachvermittlungsmethoden innerhalb des Projekts „Bilinguale Bildung - Französisch im Kindergarten" ausbildeten. Mit Blick auf das Alter, die entwicklungspsychologischen Voraussetzungen der noch sehr jungen Lerner sowie die jeweiligen Rahmenbedingungen bilingualer Bildung stellte sich zugleich die Frage nach dem Erwerbstyp: Wird im Kindergarten eine zweite Sprache - analog zur Muttersprache unbewusst erworben oder eine Fremdsprache früh gelernt? Dies steht in direktem Zusammenhang mit den sprachlichen Kompetenzen, die die Kinder bei der vorschulischen Fremdsprachenvermittlung ausbilden. Sind diese Kenntnisse und Fertigkeiten imitativ geprägt oder kommt die zweite Sprache im Sinne authentischer Kommunikation flexibel (adressaten-/themen-/ situationsangemessen) zur Anwendung?

Welche Struktur weisen die kindlichen Äußerungen auf (Satzlänge, Wortschatz/Domänen, Satzkomplexität)? In welchem Umfang bilden Kinder Sprachaufmerksamkeit aus?

Um am Ende begünstigende Faktoren bestimmen zu können, musste der Fokus der Untersuchung auch auf dem Sprachentwicklungsprozess liegen. In diesem Zusammenhang war zu erforschen, wie sich das - langfristige - Verhältnis von Rezeption und Produktion gestaltet. Welche (aus dem Erstspracherwerb bekannten) Phasen lassen sich beim Aufbau des zweiten Sprachsystems beobachten?

Welche Sprachverarbeitungsstrategien (Füllen lexikalischer Lücken, Zwischengrammatiken, Wissenstransfer) werden erkennbar? Welche Rolle spielt dabei die Erstsprache?

Die Auswahl der vier an der Untersuchung beteiligten Kindergärten erfolgte nicht nach dem Zufallsprinzip. Letztlich macht eine Studie wie diese nur Sinn in einer Einrichtung, in der die Kindergartenleitung und die Erzieherinnen der Untersuchung sowie deren Ergebnissen (positiven wie negativen) offen gegenüber stehen. Eng damit verbunden ist auch die Bereitschaft der Kindergärtnerinnen, das eigene pädagogische, sprachliche und methodische Vorgehen in Bezug auf ihre mit der frankophonen Bildung verbundenen Ziele zu reflektieren. Ohne ihr Einverständnis, „Beobachtung“ zuzulassen sowie weitergehende, für die Interpretation und Analyse notwendige Informationen zur Verfügung zu stellen, wäre ein Forschungsprojekt wie dieses nicht durchführbar gewesen. Zu Beginn begegneten nicht wenige Erzieherinnen der geplanten Untersuchung mit gemischten Gefühlen und hegten Vorbehalte, auf diesem Wege „kontrolliert“, „überprüft“, „,beurteilt“ zu werden. Durch Hospitationen in einzelnen Einrichtungen und persönliche Gespräche, in denen Ziele, Inhalte und Methoden des Forschungsvorhabens transparent gemacht wurden, gelang es, eine Vertrauensbasis zu schaffen und bei allen Beteiligten Neugier auf die Ergebnisse zu wecken.

Gleichzeitig mussten für die Studie mit Blick auf die Forschungsziele mehrere Kindergärten mit unterschiedlichen Sprachvermittlungsansätzen gewonnen werden. Dass sich am Ende mit zwei Angebots- und zwei Immersionsmodellen insgesamt vier Einrichtungen fanden, deren Konzeptionen früher Fremdsprachenvermittlung - bezogen auf die Zielsetzungen sowie Quantität und Qualität des zweitsprachlichen Inputs - ein breites Spektrum bilingualer Bildung darboten, birgt einen gewichtigen Vorteil: Dies lässt sowohl eine Betrachtung innerhalb der einzelnen Organisationsformen als auch einen Vergleich zwischen Angebots- und Immersionsmodell zu, und beantwortet damit letztlich die zentrale Frage der Untersuchung, unter welchen Bedingungen was erreicht wird beim frühen Fremdsprachenlernen in den jeweils spezifischen Kontexten. 
Festzuhalten ist, dass sich zum Zeitpunkt der Studie die Gestaltung der Fremdsprachenvermittlung bei den meisten Kindergärten noch ein Stück weit „auf dem Weg“ befand und somit variierte: Einerseits reflektierten die Erzieherinnen ihr pädagogisches Handeln in diesem Bereich sehr intensiv; suchten und erprobten zusätzliche methodische und inhaltliche Ideen. Andererseits veränderte sich in der einen oder anderen Einrichtung im Laufe des Jahres die Personalkonstellation und damit die Organisation der frühen Fremdsprachenvermittlung, wie die nun folgende Vorstellung der vier Kindergärten zeigen wird.

\section{Immersionsmodell 1: Kindergarten „Villa Pêle-Mêle“}

Im deutsch-französischen Kindergarten „Villa Pêle-Mêle“ arbeiten in jeder Gruppe eine deutsche und eine französische Erzieherin zusammen. Hier findet der Kindergartenalltag - wie in einer binationalen Familie - deutsch-französisch statt. Nach dem Prinzip der funktionalen Sprachentrennung spricht jede Erzieherin mit den Kindern nur ihre jeweilige Muttersprache.

Innerhalb der Gruppe findet die Kommunikation des Erzieherinnenteams stets auf Französisch statt. Obwohl die französischen Erzieherinnen im Kindergarten „Villa Pêle-Mêle" auch die deutsche Sprache beherrschen, wird den Kindern so vermittelt, dass diese „kein Deutsch sprechen“. Diese Methode und das bilinguale Setting stellen für die Kinder eine authentische Situation dar; es wird für sie natürlich, sich mit ihren Fragen, Wünschen, Feststellungen in der Zweitsprache an die französische Erzieherin zu wenden.

Was an dieser Stelle sehr einfach klingt, verlangt den Erzieherinnen im Alltag ein Höchstmaß an Konsequenz und Selbstkontrolle ab: So dürfen sie, um nicht aus ihrer Rolle herauszutreten, nicht über einen auf Deutsch erzählten Witz eines Kindes lachen oder gar darauf reagieren, wenn Eltern sie in Anwesenheit der Kinder auf Deutsch ansprechen. Die Vorgabe, dass die französische Erzieherin offiziell kein Deutsch spricht, wird von vielen Kindern als „kein Deutsch können“ interpretiert, was für die Fachkräfte teilweise einen Rollenkonflikt birgt.

Mit Vorliebe werden einzelne Aktivitäten in altersgemischten Kleingruppen angeboten: So stellt die französische Fachkraft sicher, dass bei „Verständigungsproblemen" zwischen ihr und jüngeren Kindern die Größeren helfen können. Dies schult nicht nur die sprachlichen Kenntnisse und Fertigkeiten. Die Anwendung ihres Könnens führt den Kindern den Nutzen der zweisprachigen Erziehung direkt vor Augen. Auch die zweisprachigen, deutsch-französischen Kinder werden geschickt in dieses „Helfersystem" eingebunden. Das gruppenteilige Verfahren bietet sich ferner zur inneren Differenzierung (der individuellen Förderung schwächerer bzw. besonders begabter Kinder) an.

Zu den Zielen des deutsch-französischen Kindergartens gehört es, die Schützlinge nach drei Kindergartenjahren zweisprachig aus der Einrichtung zu entlassen. Mit dem Übergang der ersten Kinder in die Grundschule, bemühte sich die Kindergartenleitung darum, dass die zweitsprachliche Bildung auf schulischer Ebene weitergeführt werden kann. Da dies vor Ort bislang nicht gegeben war (erst zum Schuljahr 2003/2004 wurde an der benachbarten Grundschule ein zweisprachiger Zug eingerichtet), entschieden sich einige Eltern, ihre Kinder in einer deutsch-französischen Grundschule im Elsass anzumelden. Nach Auskunft der Kindergartenleiterin bereitete den deutschen Schulanfängern das Lernen in der elsässischen Schule bzw. im französischen Schulsystem keine Schwierigkeiten. 


\section{Immersionsmodell 2: Kindergarten „Arc-en-Ciel“}

Im Kindergarten „Arc-en-Ciel“ war der Untersuchungszeitraum gekennzeichnet von personellen und organisatorischen Veränderungen. Zu Beginn arbeiteten in der Einrichtung neben überwiegend deutschen Erzieherinnen zwei französische Muttersprachlerinnen. Im Rahmen der Untersuchung wurde eine dauerhaft von einer französischen Erzieherin (sowie einer monolingualen deutschen Zweitkraft) betreuten Kinder beobachtet, die sich dabei abzeichnende zweitsprachliche Entwicklung dokumentiert und analysiert. Allerdings verfügte die deutsche Zweitkraft hier über keinerlei Französischkenntnisse, so dass die Kommunikation dieses Erzieherinnenteams zwangsläufig auf Deutsch stattfinden musste.

\section{Die Angebotsmodelle}

Hinsichtlich der nun folgenden Vorstellung der beiden Angebotsmodelle ist vorab anzumerken, dass die jeweils für bilinguale Bildung zuständigen Erzieherinnen über sehr gute Sprachkenntnisse verfügen. Beide haben Französisch bereits im Gymnasium gelernt und leben heute im Elsass. Über das Angebotsmodell vermitteln sie den Kindern daher auch ihre eigene Freude und Begeisterung für die französische Sprache und Kultur. Dennoch unterscheiden sich die beiden Angebotsansätze vor allem hinsichtlich sprachlicher Zielsetzungen erheblich voneinander.

\section{Angebotsmodell 1: Kindergarten „Raupe Nimmersatt““}

Im Kindergarten „Raupe Nimmersatt“ werden insgesamt 74 Kinder im Alter von einem bis sechs Jahre betreut. Was 1999 als zeitlich begrenztes Projekt mit zwölf Teilnehmern und einem sehr begrenzten frankophonen Repertoire begann, ist zu einem festen Bestandteil des Kindergartenalltags geworden: Heute dürfen alle Kinder in der Einrichtung - ihrem Alter und ihren Interessen entsprechend - vielfältige Erfahrungen mit der französischen Sprache und Kultur sammeln. Die Initiative zur Fortsetzung und Ausweitung des Französischangebots ging dabei von den Kindern aus. Bei den zum täglichen Ablauf gehörenden Treffen in altershomogenen Gruppen sind schon bei den Ein- bis Dreijährigen ein französisches Fingerspiel sowie die in der Fremdsprache gesungenen Begrüßungsund Abschiedslieder als festes Ritual nicht mehr wegzudenken.

Außerdem kommen sowohl die Schulanfänger als auch vier- bis fünfjährige Kinder täglich zum Französischangebot zusammen. Zwar gilt für das Französischprojekt grundsätzlich das Prinzip der Freiwilligkeit; allerdings sind die Kinder, die sich einmal dafür entschieden haben, dann auch zur regelmäßigen Teilnahme verpflichtet. Um möglichst alle Kinder mit der Zweitsprache zu erreichen, steht neben diesen festen Gruppen allen Interessierten die Teilnahme an einem offenen Mittagskreis frei, zu dem sich die Kinder nach Lust und Laune spontan zusammenfinden. Der wechselnden Besetzung entsprechend, ist dieses Angebot anders strukturiert als die Vormittagsgruppen, bei denen eine kommunikative Progression feststellbar ist. Das Repertoire an Liedern, Reimen, Fingerspielen ist beim offenen Mittagskreis mehrheitlich auf Imitation ausgerichtet, weniger umfangreich und relativ einfach, so dass ein (Wieder-) Einstieg jederzeit möglich ist.

Beim Angebotsansatz des Kindergartens „Raupe Nimmersatt“ steht zudem der Begegnungsaspekt sehr stark im Vordergrund. Wöchentlich finden Treffen mit Kindern des benachbarten deutsch-französischen Kindergartens statt. Darüber hinaus unterhält der Kindergarten regelmäßige Kontakte mit drei Vorschuleinrichtungen im Elsass (einer staatlichen école maternelle bei Straßburg sowie zwei ABCM-Klassen nahe Mulhouse). 


\section{Angebotsmodell 2: Kindergarten „Pusteblume“}

Im Kindergarten „Pusteblume“ machte der Anteil ausländischer Kinder zu Beginn 76\% aus (darunter keine Franzosen). Daher war zunächst die Stützung der deutschen Sprache ein wesentlicher Schwerpunkt in der Arbeit der beiden Vollzeitkräfte und einer Praktikantin, ehe bilinguale Bildung eingeführt wurde. Beim Angebotsansatz im Kindergarten „Pusteblume“ steht das „Erleben von Frankreich und der dortigen Kultur“ im Mittelpunkt. Sprachliche Aspekte sind der Begegnung, dem Austausch und gemeinsamen Unternehmungen ausdrücklich nachgeordnet; d.h. sie haben eher dienende Funktion: Die Kinder sollen sich im regelmäßigen Kontakt mit der elsässischen Partnergruppe "ein bisschen verständigen können“, so die Erzieherin. Zu Beginn fand das Französischangebot einmal wöchentlich statt. Später wurde versucht, die andere Sprache regelmäßig in den Alltag einzubetten; d.h. während des Stuhlkreises ein französisches Singspiel einzustreuen, in der anderen Sprache „nach dem Wetter zu sehen“ oder nebenbei zu wiederholen, wie man sich in der anderen Sprache vorstellt. Mit ihrer Begeisterung für das französische Angebot war die Kindergartenleiterin zunächst alleine. Ihre Kollegin stand dem eher skeptisch gegenüber. Mit der Zeit stellte sich jedoch fest, dass die Kinder an bestimmten Aktivitäten in der Zweitsprache Freude hatten. Schließlich belegte sie einen vom Träger bezahlten Französischkurs.

Entsprechend der Gruppenstruktur wird im Kindergarten „Pusteblume“ auch ein Begegnungssprachenkonzept realisiert, bei dem die im Kindergarten vertretenen Nationalitäten, Sprachen und Kulturen im Mittelpunkt stehen. Z.B. wird bei Geburtstagsfesten regelmäßig das Lied „Zum Geburtstag viel Glück“ in den Sprachen aller Kinder (Russisch, Chinesisch, Albanisch, Spanisch, Englisch, Türkisch, Italienisch, Französisch) gesungen.

Der quantitative Vergleich des frankophonen Sprachertrags in allen vier Kindergärten belegte, dass der zweitsprachliche Ertrag bei den Kindern nicht nur zwischen den übergeordneten Ansätzen (Immersion und Angebot), sondern auch innerhalb der einzelnen Modelle früher Fremdsprachenvermittlung in hohem Maße variierte. Diese Differenz trat insbesondere bei den Immersionskindergärten durch die Auswertung des zweitsprachlichen Anteils der Kinder hervor. Während in der Einrichtung „Villa Pêle-Mêle“ das Verhältnis deutscher und französischer Äußerungen ungefähr ausgewogen war, produzierten die Schützlinge des Immersionskindergartens „Arc-en-Ciel“ rund vier Fünftel ihrer Gesprächsbeiträge in der Erstsprache. Frappierend ähnlich entpuppte sich das Resultat bei der Gegenüberstellung der beiden Angebotsmodelle „Raupe Nimmersatt“ und „Pusteblume“. Während der französischsprachige Anteil der kindlichen Äußerungen im Kindergarten „Raupe Nimmersatt“ $17 \%$ ausmachte, betrug er in der zweiten Einrichtung, dem Kindergarten „Pusteblume“ gerade einmal $2 \%$. Die tiefergehende qualitative Analyse der kindlichen Sprachdaten lieferte Anhaltspunkte, welche Faktoren sich auf das frühe Fremdsprachenlernen im Kindergarten günstig bzw. hemmend auswirken. So sprechen die Diskrepanzen im Ergebnis der beiden Immersionsmodelle dafür, dass die individuellen Bedingungen (hier: Organisation und Methodik) der frühen Fremdsprachenvermittlung den sprachlichen Ertrag der Kinder wesentlich mitbestimmen. Unterschiede in den sprachlichen Resultaten der Angebotsansätze lassen sich außerdem durch die jeweils mit der frühen Fremdsprachenvermittlung verbundenen Zielsetzungen erklären.

Wenngleich der kindliche Zweitsprachertrag (Art und Umfang des Wortschatzes, Satzlänge Satzkomplexität, Pragmatik) in jeder der vier Einrichtungen seine ganz eigene Beschaffenheit aufweist, ergeben sich durchaus Berührungspunkte zwischen je einem Immersions- und Angebotsmodell, die sich aus dem Gesamtbild als besonders erfolgreich herauskristallisierten: In diesen Kindergärten zeugten die Sprachdaten der Kinder 
von einer erhöhten Sprechbereitschaft und einem über die im Angebotsansatz stark vertretene Imitation hinausgehenden Bemühen, mit einem (noch) begrenzten Repertoire an Redemitteln in der anderen Sprache zu kommunizieren. Dies belegen Paraphrasen, Sprachmischungen, Code switching, freie Wortschöpfungen, Lautmalereien und nonverbale Strategien (z.B. etwas zeigen, gestikulieren). Der jeweilige Duktus der kindlichen Äußerungen steht dabei in enger Abhängigkeit zum zweitsprachlichen Input.

Generell ist festzuhalten, dass die Kinder in allen vier an der Untersuchung beteiligten Einrichtungen über ausgeprägte rezeptive Fertigkeiten verfügen. Selbst die mancherorts überwiegenden deutschen Äußerungen sowie das nonverbale Agieren der Kinder haben immer wieder erkennen lassen, dass sie mit dem Kindergartenalltag verbundene französische Gesprächsroutinen der Erzieherin weitgehend verstehen. Dies erinnert beim Angebotsansatz an die aus dem Erstspracherwerb bekannte Entwicklungsabfolge „Rezeption vor Produktion“, könnte im Kontext eines Immersionsmodells aber auch als „rezeptiver Bilinguismus“ interpretiert werden. Es ist daher zu vermuten, dass in Einrichtungen, in denen die Kinder sich weniger häufig auf Französisch geäußert haben, durchaus ein passiver Wortschatz in der Zweitsprache angelegt ist und sich möglicherweise für das künftige schulische Fremdsprachenlernen als gewinnbringend erweist. Das Spielen mit der Sprache sowie das Reflektieren über Sprache zeugen von einer aktiven Auseinandersetzung mit dem Französischen sowie sich anbahnender Sprachaufmerksamkeit. Ferner zeigten die Kinder in allen Kindergärten die Bereitschaft, sich auf Unbekanntes einzulassen. Über das Erraten einzelner Wortbedeutungen in der anderen Sprache erwerben sie schrittweise die Fertigkeit, Sinnzusammenhänge aus dem Kontext zu erschließen oder zu antizipieren. Gleichzeitig erlangen sie damit eine Schlüsselkompetenz für das weitere Sprachenlernen.

Aktiv gebrauchter zweitsprachlicher Wortschatz und Redemittel orientieren sich im Kindergarten an Themen, die den Alltag (Rituale) der Kinder sowie deren Lebensbezüge kennzeichnen (Kleidungsstücke, Essen, Familie, Farben, Tiere). Dabei verfügen Kinder aus den Immersions- und Angebotsansätzen offenbar zunächst über einen gemeinsamen Wissenskern, der sich jedoch - je nach Sprachvermittlungskonzept, Quantität und Qualität des Inputs - um zusätzliche Wortfelder und Sprachstrukturen ausdifferenziert, erweitert und idealerweise im Laufe des schulischen Fremdsprachenlernens kontinuierlich ausgebaut wird.

Die aus zwei Einzelfallstudien gewonnenen Befunde zeichnen jeweils das „Profil“ einer potenziellen zweitsprachlichen Entwicklung und markieren damit einen zentralen Unterschied zwischen Immersions- und Angebotsansatz.

Timons Daten (Immersionsmodell) weisen starke Schwankungen auf, was auf einen natürlichen Erwerbsverlauf mit Sprüngen und Schüben hindeutet. Insgesamt verfügte Timon über ein breites zweitsprachliches Repertoire; d.h. einen vielseitigen Wortschatz insbesondere im Bereich der Alltagskommunikation sowie eine zunehmend komplexe Syntax. Seine Strategien zum Füllen semantischer Lücken (z.B. Paraphrasen, Onomatopoetika, kreative Wortschöpfungen) zeigen seine Bereitschaft, trotz sprachlicher Barrieren in der Zweitsprache zu bleiben statt auf die Erstsprache auszuweichen. Zugleich war bei ihm als weiteres Anzeichen einer sich anbahnenden ausgewogenen Zweisprachigkeit ein auf Themen, Adressaten und Situationen bezogenes Code Switching zu beobachten.

Die Auszählung von Valeries gesamtsprachlichen Korpus (Angebotsmodell) beschreibt einen linearen Prozess wie beim Sprachenlernen nach einem vorab festgelegten Curriculum. Zwar lässt sich hieraus quantitativ der langsame, sukzessive Ausbau von Wortschatz, einfachen Strukturen und Redeakten ablesen; der Begriff einer 
linguistischen Progression würde in diesem Zusammenhang jedoch zu weit führen. Zu Beginn der Untersuchung kennzeichnen ein thematisch eng abgesteckter Wortschatz, ein imitativ-reproduktiver Sprachgebrauch sowie ritualisierte Redeakte Valeries sprachliches Korpus. Paraphrasen oder französisch dominierte Sprachmischungen wie bei Timon fehlen hier zunächst ganz. Im Untersuchungszeitraum veränderte die Erzieherin die Struktur ihres Sprachangebots; versuchte mit den Kindern mehr auf Französisch zu kommunizieren. Dies bildet sich auch in Valeries Entwicklung ab, indem zunehmend zweitsprachliche Wortschöpfungen sowie ein zielgerichteter spielerischer Umgang mit der Sprache im Sinne eines Bemühens um zweitsprachliche Kommunikation zu beobachten sind.

Das Ergebnis besagt indes: In Abhängigkeit von den organisatorischen, inhaltlichen und methodischen Gestaltungsmöglichkeiten konnte das Angebotsmodell im Kindergarten „Raupe Nimmersatt" deutlich mehr als „nur" sensibilisieren. Allerdings wird es kaum zu ausgewogener Zweisprachigkeit führen, wie sie sich das Immersionsmodell auf die Fahnen geschrieben hat. Dafür sind zusätzliche Eckdaten notwendig, die sich aus dem Vergleich der beiden Einzelfallstudien eindeutig herauskristallisierten.

Anhand der Einzelfallstudien sollten eigentlich die Unterschiede zwischen Immersions- und Angebotsmodell herausgearbeitet werden. Bei vertiefter Auseinandersetzung mit dem Datenmaterial der Kinder stellte sich jedoch heraus, dass Valeries Entwicklung sich in manchen Bereichen zaghaft dem annäherte, was man vielmehr mit dem Immersionsmodell in Verbindung bringt: Das Bestreben, sich in der Zweitsprache auszudrücken bis hin zum Versuch, (selbst mit einem relativ begrenzten Repertoire) auf Französisch zu kommunizieren. Insofern steht ihre Einzelfallstudie hier für die mit dem Angebotsansatz verbundenen Möglichkeiten und Chancen. Denn anders als das Immersionsmodell, dessen Effizienz durch die Erkenntnisse aus der Bilinguismusforschung wissenschaftlich kaum angezweifelt wird, bedarf die frühe Fremdsprachenvermittlung im Kindergarten über den aus sprachdidaktischer Sicht bis dato doch eher unorthodoxen Angebotsansatz der Legitimierung. Einen Beitrag dazu kann das vorliegende Forschungsergebnis leisten, wenn es sich durch weitere Einzelfallstudien bzw. Untersuchungen mit einer größeren Stichprobe untermauern lässt.

\section{Frühes Fremdsprachenlernen im Kindergarten: Gelingensbedingungen}

Aufgrund der bisherigen Befundlage ist davon auszugehen, dass folgende Faktoren im Wesentlichen das Gelingen der frühen Fremdsprachenvermittlung im Projekt „Bilinguale Bildung - Französisch im Kindergarten" mitbestimmt haben:

- Die Erzieherin als Sprachmodell und Vermittlerin der anderen Sprache und Kultur

- Die Kinder mit ihrer entwicklungspsychologischen Disposition

- Der (angemessen ausgewählte) Sprachvermittlungsansatz mit seiner spezifischen Methodik

- Quantität und Qualität des Inputs ausgerichtet auf die jeweiligen Ziele

- Eltern und Kindergartenträger als erweiterter Rahmen

Zunächst beziehen sich die genannten Einflussgrößen ganz allgemein auf die frühe Fremdsprachenvermittlung in den untersuchten Kindergärten, unabhängig von ihrer jeweils übergeordneten methodischen Ausrichtung (Sprachbad bzw. zeitlich begrenztes Angebot).

Gleichwohl muss in Teilbereichen gelegentlich zwischen den Immersions- und Angebotsansätzen differenziert werden. Sofern dies jedoch nicht ausdrücklich angemerkt ist, beziehen sich die nun folgenden Erläuterungen auf beide Konzeptionen. 
Wie der Erstspracherwerb ist auch das frühe Fremdsprachenlernen als ein interaktiver Prozess zu sehen; d.h. das Angebot und die Anregung durch die (zweit)sprachliche Umgebung entscheiden maßgeblich darüber, was und wie viel die Kinder sich vom Französischen aneignen können. Dieses zweitsprachliche Umfeld repräsentieren und gestalten im Kindergarten einzelne Erzieherinnen. Über den frankophonen Input und ihr methodisches Handeln steuern sie letztlich die Effektivität der frühen Fremdsprachenvermittlung. Den Erkenntnissen der Bilinguismus- bzw. der Lernforschung entsprechend beeinflusst darüber hinaus das (emotionale) Verhältnis der Erzieherin zu den Kindern ganz entscheidend, wie viel diese von ihr als Sprachmodell bzw. Vermittlerin der anderen Sprache und Kultur annehmen.

Ihre Vorbildfunktion beschränkt sich indes nicht alleine auf die korrekte Anwendung der Zweitsprache. Ebenso wichtig ist ihre persönliche Haltung zur bilingualen Bildung. Nur wenn sie hinter dieser Aufgabe steht, sie freudig und mit einer gewissen Ernsthaftigkeit ausführt, kann sie ihre Schützlinge dauerhaft motivieren und Lerneffekte erzielen. Daher sollte sich die pädagogische Fachkraft genau im Klaren sein darüber, welche Ziele sie mit der frühen Fremdsprachenvermittlung verfolgt und ihre Methodik danach gestalten: Im Idealfall richtet sie beides nach ihren eigenen Möglichkeiten (u.a. Sprachkompetenz), den Gegebenheiten im Kindergarten und nicht zuletzt auch an den Bedürfnissen der Kinder aus. Indirekt beeinflussen auch die Berufsauffassung der Erzieherin und ihr Bild vom Kind (was und wie viel traut sie ihm zu?) ihre mit der frühen Fremdsprachenvermittlung verbundenen Ziele. Letztere schlagen sich auf das sprachliche und methodische Handeln der Erzieherin nieder; d.h. sie entscheiden somit wesentlich darüber, wie viel Französisch an die Kinder herangetragen wird.

Im Gegenzug dazu nehmen Kinder offenbar sehr schnell intuitiv zur Kenntnis, was von ihnen (nicht) verlangt wird. Über die Gestaltung der bilingualen Bildung sendet die Erzieherin stille Botschaften aus. Durch ständiges Übersetzen der eigenen oder kindlicher Äußerungen bzw. dauerhaftes Akzeptieren deutscher Antworten auf französische Fragen oder die explizite Feststellung „es muss nicht unbedingt Französisch gesprochen werden" entfällt für die Kinder die Notwendigkeit (und zugleich auch die Chance!), sich in der Zweitsprache zu verständigen. Die Zahlen aus der vorangegangenen Studie scheinen zu belegen, dass der frankophone Sprachertrag der Kinder gesteigert werden kann bei einem Sprachmodell, das beim Französischen bleibt und die Kinder - wie beim Erstspracherwerb - z.B. durch häufiges Nachfragen zur Verwendung der Sprache herausfordert.

Durch die nach der PISA-Studie entbrannte Diskussion um eine angemessenere Förderung bereits im Vorschulalter bahnt sich im Elementarbereich und unter den Erzieherinnen zunehmend ein neues Selbstverständnis an, das sich u.a. in der Einführung von Projekten wie dem frühen Fremdsprachenlernen im Kindergarten niederschlägt: Der bisherige Fokus der Kindergartenpädagogik (soziales Lernen, Betreuung, kreatives Tun) wird um kognitiv ausgerichtete Bildungsinhalte erweitert. Um mit diesen Entwicklungen Schritt halten zu können, bedarf es flächendeckender Möglichkeiten zur systematischen Weiterqualifizierung sowie einer Umstrukturierung der Ausbildung (in Anlehnung an andere europäische Länder z.B. Frankreich), einhergehend mit einer Aufwertung des Berufsstands.

Die Arbeit im Elementarbereich hat auch bislang fundierte Kenntnisse zur Entwicklungspsychologie und zum Spracherwerb bzw. Sprachförderung vorausgesetzt, so dass eigentlich jede Erzieherin nach einer soliden Fortbildung in der Lage sein sollte, zweitsprachliche Aktivitäten auf der Basis von Sensibilisierung - mit Liedern, Reimen und Fingerspielen - anzubieten. Strebt sie darüber hinausgehende Sprachvermittlung z.B. analog zum Angebotsmodell des Kindergartens „Raupe Nimmersatt“ an, sollte sie über eine angemessene Sprach- und Methodenkompetenz verfügen. 
Als erste Epoche der Gesamtentwicklung stellt das Kindergartenalter einen bedeutsamen Lebensabschnitt dar, in dem auf sprachlicher, kognitiver und sozial-emotiver Ebene viele Grundlagen geschaffen werden. Der Begriff „Breitenförderung“ trifft durchaus den Kern des frühen Fremdsprachenlernens im Kindergarten. Denn bilinguale Bildung im Kindergarten stellt einerseits für die leistungsstarken Vorschüler das von der "Gesellschaft für das hochbegabte Kind“ dringend angemahnte „Futter für's Gehirn“ dar. Gleichzeitig finden aber auch im Sinne der Chancengleichheit Kinder Zugang zur französischen Sprache und Kultur, denen das sonst von Haus aus höchstwahrscheinlich verwehrt bliebe. Kinder können nachweislich mit mehr als einer Sprache aufwachsen: Wird bilinguale Bildung fundiert umgesetzt, stellt sie weder eine Überforderung noch einen schädlichen Einfluss (z.B. hinsichtlich der Erstsprache) dar. Es ist jedoch im Auge zu behalten, dass Kinder - je nach Persönlichkeit und individueller Begabung - unterschiedlich mit dem zweitsprachlichen Angebot umgehen; daher auch nicht notwendigerweise in gleicher Art und gleichem Umfang davon profitieren.

Grundsätzlich gilt es, bei der Gestaltung der frühen Fremdsprachenvermittlung natürliche, altersspezifische Potenziale der Kinder gewinnbringend zu nutzen. Zu den günstigen Voraussetzungen, die Vorschulkinder für den frühen Zweitspracherwerb mitbringen, gehören neben ihrer Anpassungsfähigkeit, Unvoreingenommenheit, Offenheit, Spontaneität vor allem ihre (oft unterschätzte) Lernfreude und Leistungsbereitschaft. Die meisten Kinder wollen nicht nur spielen, sondern streben nach eigenen greifbaren Spracherfahrungen. Sofern ihnen dazu Raum gelassen wird, erproben sie frei von der Angst vor Fehlern oder unbekannten Strukturen sprachliche Muster in einem höchst kreativen Umgang mit der Zweitsprache. Dabei hat sich der Aspekt der Begegnung bzw. des Sprachhandelns mit frankophonen peers (als Sprachmodell und Kommunikationspartner) innerhalb des eigenen Kindergartens als wertvoll erwiesen.

Insofern scheint die Untersuchung zu belegen, dass eine moderate Ergebnisorientierung für Kinder eine Motivation darstellt. Zu viel Beliebigkeit und Unverbindlichkeit bescheren Kindern hingegen kaum Erfolgserlebnisse oder das Gefühl, in ihrem Tun ernst genommen zu werden.

Bei (nicht frankophon) bilingualen Kindern bestätigten sich die im ersten Teil der Arbeit erläuterten Konzepte der erhöhten Sprachaufmerksamkeit bzw. flexibleren Handhabung weiterer sprachlicher Systeme: Von Haus aus zweisprachige Kinder fanden auffallend schnell Zugang zur Drittsprache und zeigten besonderes Geschick im Umgang mit dem französischen Lautsystem, Wortschatz und Sprachstrukturen. Aus der Untersuchung geht jedoch hervor, dass auch zunächst monolinguale Kinder - je nach Anlage und Förderung durch ihr Umfeld - durchaus vergleichbare Potenziale besitzen.

Die Befunde der Untersuchung geben außerdem Aufschluss darüber, wie die Quantität und Qualität des Inputs für eine gewinnbringende Fremdsprachenvermittlung im Kindergarten beschaffen sein sollten. Unabhängig von einer bestimmten methodischen Konzeption hat sich dabei die Natürlichkeit des jeweiligen Settings als zentraler Punkt herauskristallisiert. Hinsichtlich des zweitsprachlichen Ertrags bei den Kindern haben sich nämlich jene Einrichtungen als besonders erfolgreich erwiesen, in denen das Französische idealerweise täglich und in einem ganzheitlich-kommunikativen Kontext dargeboten wurde.

Das Ergebnis der Studie verschiedener Gruppen legt nahe, dass sich der Anteil der französischsprachigen Äußerungen seitens der Kinder in Abhängigkeit vom Sprachverhalten der Erzieherin entwickelt. Indem sie die französische Sprache in den Mittelpunkt stellt, entsteht für die Kinder ein Anreiz, sich ebenfalls in der Zweitsprache 
auszudrücken und mitzuteilen. Bei der Analyse der kindlichen Sprachdaten haben sich die Bereitschaft und Disziplin der Kinder, in der Zweitsprache zu bleiben als wichtiger Faktor für das Gelingen der frühen Fremdsprachenvermittlung herausgestellt.

Durch ihren Sprachgebrauch liefert die Erzieherin als Sprachmodell den Kindern im Bereich des Wortschatzes, der Syntax und der Pragmatik (verfeinerte) Muster. Durch die Konsequenz in der Sprachwahl erhöht sich automatisch die Quantität des frankophonen Inputs, dessen Idealmaß - wohl in allen Bereichen des Spracherwerbs - auf die einfache Formel „je mehr und je vielfältiger, umso besser“ gebracht werden kann. Vor diesem Hintergrund ist es gewiss erstrebenswert, für die bilinguale Bildung im Kindergarten verstärkt französische Muttersprachlerinnen zu gewinnen, da diese eine flexiblere und nuanciertere Sprachverwendung an die Kinder herantragen können.

Trotzdem sehe ich ein muttersprachlich geprägtes Modell nicht als alternativenlose Grundbedingung für die frühe Fremdsprachenvermittlung im Kindergarten. Zumal die Untersuchungsergebnisse darauf hindeuten, dass die Sprachvermittlung durch eine Muttersprachlerin nicht zwangsläufig eine Erfolgsgarantie darstellt. Auch eine deutsche Erzieherin mit sehr guten Französischkenntnissen und dem notwendigen methodischen know-how kann Beachtliches erreichen, wie das Datenmaterial aus dem Kindergarten „Raupe Nimmersatt“ gezeigt hat. Grundsätzlich gilt bei allen Aktivitäten im Bereich der bilingualen Bildung (auch bei Sensibilisierung!) Sprachrichtigkeit als absolutes Muss; d.h. je mehr sprachliche Kenntnisse und Fertigkeiten bei den Kindern angezielt werden, umso höhere Sprachkompetenz sollte bei der Erzieherin vorhanden sein.

Wie aus den Theorien des Erst- und Zweitspracherwerbs hervorgeht, lernen die Kinder die Sprache ihrer Umgebung durch an sie gerichtete Äußerungen. Die Auswertung der Transkripte spiegelte deutlich bestimmte Strukturen in der Erzieherinnensprache wieder, wie sie aus wissenschaftlicher Sicht für den Spracherwerb als förderlich angenommen werden. So verwendete die Muttersprachlerin im Immersionsmodell der „Villa Pêle-Mêle" häufig Fragen, Imperative, arbeitsbegleitendes Sprechen und Paraphrasen. Während Imperative und (Rück-)Fragen die Kinder sprachlich oder nonverbal (z.B. Ausführen von Anweisungen) aktivieren, reichern das arbeitsbegleitende Sprechen und die Paraphrasen das Sprachrepertoire der Kinder an. Vor allem die Umschreibungen sind nicht nur auf der Wortschatzebene für den Aus- und Umbau des mentalen Lexikons sehr wichtig; sie stellen zugleich sprachliche und soziokulturelle Muster für einen verfeinerten Sprachgebrauch dar und vertiefen somit auch in der Zweitsprache die interaktionale Kompetenz der Kinder. Die Paraphrasen der Muttersprachlerin können zugleich als ein Element des motherese interpretiert werden. In diesem Rahmen kommt der Reformulierung die Bedeutung einer bewussten mütterlichen Korrekturstrategie zu: Bei der Beobachtung im Immersionskindergarten entstand ebenfalls der Eindruck, dass die Erzieherin die Reformulierungen sehr gezielt verwendete und so - wie Mütter gegenüber älteren Kindern - auch explizit die Rolle der Sprachlehrenden einnahm.

Diese finden wir bei der Sprache der Erzieherin aus dem Angebotsmodell seltener, da der von ihr an die Kinder gerichtete frankophone Input anders strukturiert ist. Ihr Sprachhandeln ist einerseits offenbar von eigenen (schulischen) Sprachlernerfahrungen geprägt. Gleichzeitig trägt es Züge des motherese und ist methodisch auf den natürlichen, in einer frühen Phase befindlichen Spracherwerbsprozess ausgerichtet: Ein begrenzter kindgemäßer Wortschatz und einfache Sprachstrukturen wiederholen sich in wechselnden Handlungskontexten (u.a. Lieder, Spiele, Reime = ritualisierte 
Wortspiele). Dabei wird das Prinzip des Sprachüberflusses wirksam. Im Bereich der Artikulation tritt das mütterliche Register gelegentlich durch deutliches, langsameres Sprechen, Pausen an Wort- oder Satzenden sowie eine höhere Stimmlage in Erscheinung. Nachweislich dient der (meist intuitive) Gebrauch des motherese der Herstellung eines affektiven Bezugs zwischen Mutter (hier: der Erzieherin) und den Kindern. Daher verwundert es auch nicht, dass vor allem die erhöhte Stimmlage und beiläufige Korrekturen („Shaping“, z.B. Expansion) bei beiden Erzieherinnen, sowohl im Immersions- als auch im Angebotsmodell, zu beobachten waren. Die von der Erzieherin im Immersionsansatz verwendeten mütterlichen Sprachlehrstrategien kommen der aktuellen kognitiven und sozialen Entwicklung der Kinder nahe, während die ihrer Kollegin aus dem Angebotsmodell auf eine frühere Phase zurückgehen. Dies ist aufgrund der methodischen Konzeption früher Fremdsprachenvermittlung (der Lernsituation der Kinder, der Quantität des Inputs) angemessen, bildet sich aber auch in deren zweitsprachlicher Entwicklung ab.

Will frühe Fremdsprachenvermittlung der kognitiven, sozialen und emotionalen Entwicklung der jungen Lerner gerecht werden, muss diese in erster Linie nach elementarpädagogischen Grundsätzen gestaltet werden: Neben musischen und spielerischen Aktivitäten stehen dabei idealerweise Themen und Inhalte im Vordergrund, die den Kindern die andere Sprache und Kultur auf ganzheitlichem Weg (mit allen Sinnen und auf affektiver Ebene) näher bringen. Aus lernpsychologischen Gründen, insbesondere für die Organisation des mentalen Lexikons kommt der Vernetzung von Sprache und Handlung zentrale Bedeutung zu. Regelmäßige Wiederholungen zur Festigung und Vertiefung des Inputs sind unerlässlich. Diese Maxime fand auch beim Angebotsansatz des Kindergartens „Raupe Nimmersatt“ Berücksichtigung, wo den Kindern französische Vokabeln und Sprechakte aufgrund ständiger Wiederkehr nahe gebracht wurden.

Den entwicklungspsychologischen Voraussetzungen dieser Altersstufe entsprechend sollte sich die frühe Fremdsprachenvermittlung im Kindergarten an den Grundmustern des Erstspracherwerbs orientieren, die beim Aufbau eines zweiten Systems wirksam werden. Dadurch entsteht eine Schnittstelle zwischen den scheinbar grundverschiedenen Konzeptionen von Immersions- und Angebotsmodell(en).

Die Erstsprache wird dialogisch, in einem sozialen Kontext, erworben. Unter dieser Prämisse scheint es angebracht, den Aufbau des zweiten Sprachsystems und interaktionaler Kompetenz so oft wie möglich über authentische Gesprächssituationen zu unterstützen. Während die konsequente Einsprachigkeit nach dem Prinzip der funktionalen Sprachentrennung (i.d.R. „une personne - une langue“) im Immersionsmodell als eine Grundbedingung für Sprachentrennung und einen erfolgreichen Zweitspracherwerb angesehen werden muss, wäre es jedoch verfehlt, dies auf ein Angebotsmodell zu übertragen. Da der Input das Ergebnis der Fremdsprachenvermittlung bestimmt, macht es zwar Sinn, wenn auch eine deutsche Erzieherin mit guter Sprachkompetenz im Rahmen eines Angebotsmodells möglichst viel Französisch mit den Kindern spricht. Dabei muss sie sich aber bewusst sein, dass sie eine andere Rolle einnimmt als die Muttersprachlerin, die bei einem Immersionsansatz als Repräsentantin der französischen Sprache und Kultur auftritt.

Die Anbahnung einer zweitsprachlichen Kommunikation zwischen Erzieherin und Kind vollzieht sich demnach im Angebotsansatz in einem anderen quantitativen und qualitativen Umfang als beim Sprachbad. Die Erzieherin im Angebotsansatz sollte dabei sehr behutsam vorgehen, indem sie z.B. zunächst immer wiederkehrende Anweisungen und (Handlungs-)Kommentare ausschließlich auf Französisch äußert. Mit zunehmender Sprachkenntnis der Kinder gilt es, bei diesen ein Mitteilungsbedürfnis 
zu wecken und sie z.B. in Entscheidungsprozesse sowie die Gestaltung frankophoner Aktivitäten einzubinden. Zugleich erfordert dies, den Kindern - idealerweise induktiv über das eigene Sprachverhalten - entsprechende Redemittel bereitzustellen. So wie die Muttersprachlerin im Immersionsmodell (analog zur Mutter, die ihr Kind beim Erstspracherwerb unterstützen möchte) ihre Äußerungen zu Beginn an Wahrnehmungen und visuelle Stützen koppelt, kann auch die Erzieherin im Angebotsmodell anfangs Erst- und Zweitsprache ergänzend verwenden. Mit der Zeit steigert sie den Anteil der frankophonen Äußerungen, während die erstsprachlichen langsam zurückgehen.

Auch Bilderbücher eignen sich - unabhängig vom methodischen Ansatz - für den Aufbau eines zweiten Sprachsystems bei Kindern. Erzieherinnen aus dem Angebotsmodell verwenden idealerweise Geschichten mit weniger komplexem Inhalt und vereinfachen den Erzähltext. Die Illustrationen erleichtern den Kindern als visuelle Stützen außerdem das Verständnis. Bilder und Geschichten berühren auf der emotionalen Ebene und fordern so zum Sprechen heraus; dies kann methodisch genutzt werden, um bei den jungen Lernern die Herausbildung alternativer Ausdrucksfähigkeiten (schöpferischer Umgang mit der Zweitsprache, nonverbales Agieren zum Überbrücken lexikalischer Lücken) fördern.

Diese Anregungen mögen für die frühe Fremdsprachenvermittlung im Kindergarten für einen Angebotsansatz recht hochgegriffen anmuten. Dabei gilt aber die Devise: Die Sprachanregung regelt zwar den Sprachertrag, versteht sich aber als ein offenes Angebot. D.h. Letztlich entscheiden die Kinder selbst, ob sie es annehmen möchten oder nicht. Falsch wäre es m.E. jedoch, ihnen diese Chance von vorneherein vorzuenthalten.

Bei meinen Beobachtungen im Kindergarten traten sie nur marginal in Erscheinung - und doch beeinflussen die Eltern als erweiterter Rahmen der frühen Fremdsprachenvermittlung indirekt deren Ergebnis. Ihre Einstellungen und ihr Verhalten prägen das Kind und wirken sich positiv oder negativ auf den Lernprozess aus. Zugleich bestimmt ihr sozioökonomischer Status mit darüber, ob sie ihrem Nachwuchs Zugang zu fremdsprachlicher Bildung gewähren können und wollen.

Die Bilinguismusforschung betont im Kontext der häuslichen Zweisprachigkeitserziehung die Wertschätzung beider Sprachen und Kulturen als unerlässliche Voraussetzung für ein Gelingen. Eben diese Anerkennung kommt zum Ausdruck, wenn Eltern mit ihren Kindern nach Frankreich in Urlaub fahren, selbst Französisch lernen oder die Erzieherin bei Unternehmungen z.B. im Rahmen der Begegnung unterstützen. Meine Beobachtungen im Kindergarten haben gezeigt, dass viele Eltern der bilingualen Bildung ihrer Kinder reges Interesse entgegen bringen. Daher ist es von Vorteil, sie in die frühe Fremdsprachenvermittlung einzubinden, sei es durch ihre Teilnahme an Aktivitäten oder über einen Elternbrief, der u.a. Liedtexte und Spielideen enthält. Das darf jedoch nicht darin münden, dass die Erwachsenen ihre Kinder regelrecht Vokabeln abfragen oder zu Hause ständig zum „Üben“ anhalten. Übertriebener Ehrgeiz wirkt sich hier eher schädlich aus, wie aus den Erkenntnissen der Bilinguismusforschung hervorgeht: Kinder wollen nicht auf ihre Zweisprachigkeit „getestet“ werden. Daher ist Eltern dringend zu raten, die zweitsprachliche Entwicklung ihrer Kinder - analog zum Erstspracherwerb - mit Interesse, Geduld und liebevoller Aufmerksamkeit zu begleiten. Das bedeutet ganz konkret, dem Nachwuchs dessen individuelles Tempo zuzugestehen und stille Phasen zu respektieren.

Aufgrund der Beschreibungen der beiden übergeordneten Sprachvermittlungskonzeptionen (Immersion/Sprachbad und zeitlich begrenztes zweitsprachliches Angebot) innerhalb des Projekts „Bilinguale Bildung - Französisch im Kindergarten“ 
mag der Eindruck einer Polarisation entstanden sein. So wird das Angebotsmodell zunächst eher dem spielerischen frühen Sprachenlernen (unter Berücksichtigung der Erstspracherwerbsmechanismen) zugeordnet, während für das Immersionsmodell ein unbewusster, natürlicher (mit dem Erstspracherwerb nahezu gleichgesetzter) Prozess charakteristisch zu sein scheint: Imitation versus Anwendung; Spiel versus "echte“ Kommunikation.

Das Ergebnis der vorliegenden Untersuchung deutet darauf hin, dass diese Trennung so klar nicht existiert. Ritualisierte Sprachspiele, Lieder und Reime, die sonst eher mit dem Angebotsansatz assoziiert werden, kamen auch im Immersionskindergarten regelmäßig als integraler Bestandteil der Sprachförderung zum Einsatz. Beim Angebotsmodell hingegen entstanden gelegentlich Situationen, die als „Minikommunikation" (sowohl zwischen der Erzieherin und den Kindern als auch den Kindern untereinander) bezeichnet werden können. Folglich sind das Wirksamwerden mütterlicher Sprachlehrstrategien sowie zweitsprachliche Kommunikation nicht alleine dem Immersionsmodell vorbehalten, sondern treten - in unterschiedlicher Quantität und Qualität - bei beiden Ansätzen der frühen Fremdsprachenvermittlung in Erscheinung. Beiden übergeordneten Konzeptionen der bilingualen Bildung (Immersion/Angebot) ist dies umso mehr angemessen, da die entwicklungspsychologischen Voraussetzungen der Kinder eher für einen Spracherwerb als für ein Sprachenlernen sprechen.

Das Angebotsmodell des Kindergartens „Raupe Nimmersatt“ nimmt dabei eine Art „Sandwich“-Stellung ein: Die Auswertung des Datenmaterials ergab, dass der zweitsprachliche Ertrag der Kinder, die sich quasi selbst sprachliche Ziele setzten, in dieser Einrichtung über Sensibilisierung hinausgeht. Wenngleich zwischen Angebotsund Immersionsmodell in einigen Bereichen eine Annäherung erkennbar war, traten im Vergleich der sprachlichen Daten grundlegende Unterschiede zutage: In den Ergebnissen beider Ansätze, die hier an zwei Einzelfallbeispielen dokumentiert wurden, bildet sich wie in einem Spiegel die Beschaffenheit des Inputs (und auch der Methodik) ab: Demnach wurden im Immersionsmodell ein vielseitigerer Wortschatz, eine komplexere Syntax und insgesamt ein breiteres sprachliches Repertoire vermittelt als im Angebotsansatz. Timons Entwicklung weist Schwankungen auf, erreicht aber ein höheres Niveau als Valerie, deren zweitsprachliche Kenntnisse und Fertigkeiten langsam linear, insgesamt doch weniger steil ansteigen. Dennoch zeichnet sich bei dem Mädchen aus dem Angebotsansatz des Kindergartens „Raupe Nimmersatt“ hinsichtlich ihres Erwerbs- bzw. Lernprozesses am Ende der Untersuchung eine ähnliche Entwicklungstendenz wie bei Timon ab. Aufgrund der unterschiedlichen Vermittlungsformen und letztlich durch die voneinander abweichende Struktur des Inputs vollzieht sich dies jedoch in einem anderen sprachlichen Umfang und zeitlichen Ablauf.

Diese sanfte Annäherung zwischen den übergeordneten methodischen Ansätzen macht letztlich das Erfolgsgeheimnis der frühen Fremdsprachenvermittlung im Kindergarten „Raupe Nimmersatt“ aus. Hier hat sich die kleinschrittige Übernahme einzelner wissenschaftlich als effizient gesicherter Elemente aus dem Immersionsmodell bewährt (tägliches Französischangebot, konsequentere Verwendung der französischen Sprache durch die Erzieherin.)

Aufgrund der Befunde zum frankophonen Sprechanteil - u.a. der Zunahme der französischen Äußerungen seitens der Kinder analog zu der verstärkten Verwendung der französischen Sprache durch die Erzieherin - ist zu vermuten, dass die Ausbildung kommunikativer Fähigkeiten auch mit einem verstärkt auf pragmatische Aspekte und konkrete Handlungssituationen ausgerichteten Input einhergeht. Insofern hat es den Anschein, dass sich im Rahmen der frühen Fremdsprachenvermittlung im Kindergarten 
ein Blick über den Tellerrand hinüber zu anderen Ansätzen durchaus lohnt. Zwar sollte dies nicht zu falschen Zielsetzungen oder gar der Verwässerung von Methoden (ver-) führen. Optimale Bedingungen vorausgesetzt, kann das Angebotsmodell dennoch aufgrund der Quantität und Beschaffenheit des zweitsprachlichen Inputs nicht den gleichen Effekt haben wie ein sachgerecht ausgeführter Immersionsansatz. Dennoch können einzelne Elemente daraus maßvoll, modifiziert und didaktisch sinnvoll begründet, gewinnbringend in das Angebotsmodell einfließen. Auf diese Weise wird nicht unter falschen Voraussetzungen „künstlicher Bilinguismus“ kreiert, sondern der Angebotsansatz durch Erkenntnisse aus der Bilinguismus- und Sprachlehrforschung angemessen erweitert.

\section{Fazit}

Bilinguale Bildung ist keineswegs eine elitäre Angelegenheit, sondern kann grundsätzlich von allen Kindern (unabhängig von ihrer Herkunft oder ihren bisherigen Spracherfahrungen) bewältigt werden. Dabei muss das Sprachangebot keineswegs auf eine einfache Sprache wie Farben, Zahlen, Kinderreime, Lieder reduziert werden. Vielmehr können die Kinder auch an bildungs- und schriftsprachlich ausgerichtete literate Register herangeführt werden. Beim Vorlesen und Erzählen von Geschichten, aber auch bei der Begegnung mit fremdsprachlicher Lyrik pendeln die Kinder zwischen Mündlichkeit und Schriftlichkeit und bauen neben den alltagssprachlichen auch kognitivakademische Sprachfähigkeiten auf (siehe auch Nauwerck, 2013).

Neben den sprachlichen Lerneffekten legitimieren nicht zuletzt auch der Lerneifer und die Freude, mit der die Vorschüler an das frühe Fremdsprachenlernen herangegangen sind, die Neuausrichtung der frühkindlichen Bildung in deutschen Kindergärten.

\section{Literatur}

Cameron, L. (2001): Teaching Languages to Young Learners. Cambridge University Press, Cambridge.

Häckel, A. \& Piske, T. (2011): Zur Entwicklung der sprachlichen Fähigkeiten bei deutschenglisch bilingual betreuten Kindergartenkindern mit und ohne Migrationshintergrund. In: C. Spiegel \& A. Krafft (Hg.), Sprachliche Förderung und Weiterbildung transdisziplinär. Peter Lang, Frankfurt a. M., 11-31.

Kersten, K. et al. (2010a, Hg.): Bilingual Preschools. Vol. I: Learning and development. Wissenschaftlicher Verlag Trier, Trier.

Kersten, K. et al. (2010b, Hg.): Bilingual Preschools. Vol. II: Best Practises. Wissenschaftlicher Verlag Trier, Trier.

Nauwerck, P. (2012): Zweisprachigkeit im Kindergarten. Konzepte und Bedingungen für das Gelingen. 2. Auflage. Fillibach, Freiburg im Breisgau.

Nauwerck, P. (2013): Vorschulische Sprachförderung: Mehrsprachige Kinderliteratur als Wegbereiterin von der Mündlichkeit zur Schriftlichkeit. In: I. Gawlitzek \& B. KümmerlingMeibauer (Hg.), Mehrsprachigkeit und Kinderliteratur. Fillibach bei Klett, Stuttgart, 239-262.

Oomen-Welke, I. (2003): Entwicklung sprachlichen Wissens und Bewusstseins im mehrsprachigen Kontext. In U. Bredel et al. (Hg.), Didaktik der deutschen Sprache. Ferdinand Schöningh (UTB), Paderborn, München, Wien, 452-461.

Piske, T. (2007): Wichtig ist nicht nur ein früher Beginn: Zum Erlernen von Fremdsprachen an Kindertageseinrichtungen und Grundschulen. In: M. Plieninger \& E. Schumacher (Hg.), Auf den Anfang kommt es an - Bildung und Erziehung im Kindergarten und im Überfang zur Grundschule. (= Schwäbisch Gmünder Hochschulreihe 27). Schwäbisch Gmünd, 133-151. 
Stern E. et al. (2005, Hg.): Lehr-Lernforschung und Neurowissenschaften - Erwartungen, Befunde, Forschungsperspektiven. Bundesministerium für Bildung und Forschung (BMBF) Band 13. Bonn und Berlin.

Wehr, S. (2001): Was wissen Kinder über Sprache? Verlag Paul Haupt, Bern, Stuttgart und Wien.

Wode, H. (2009): Frühes Fremdsprachenlernen in bilingualen Kindergärten und Grundschulen. Westermann, Braunschweig. 